\title{
New Interaction Solutions of (3+1)-Dimensional KP and (2+1)-Dimensional Boussinesq Equations
}

\author{
Bo Ren, Jun Yu, and Xi-Zhong Liu \\ Institute of Nonlinear Science, Shaoxing University, Shaoxing 312000, China \\ Correspondence should be addressed to Bo Ren; renbosemail@163.com
}

Received 22 May 2015; Accepted 6 July 2015

Academic Editor: Leszek Gasinski

Copyright (C) 2015 Bo Ren et al. This is an open access article distributed under the Creative Commons Attribution License, which permits unrestricted use, distribution, and reproduction in any medium, provided the original work is properly cited.

\begin{abstract}
The consistent tanh expansion (CTE) method has been succeeded to apply to the nonintegrable (3+1)-dimensional KadomtsevPetviashvili (KP) and (2+1)-dimensional Boussinesq equations. The interaction solution between one soliton and one resonant soliton solution for the (3+1)-dimensional KP equation is obtained with CTE method. The interaction solutions among one soliton and cnoidal waves for these two equations are also explicitly given. These interaction solutions are investigated in both analytical and graphical ways. It demonstrates that the interactions between one soliton and cnoidal waves are elastic with phase shifts.
\end{abstract}

\section{Introduction}

The investigation of exact solutions of nonlinear partial differential equations (PDEs) plays an important role in the study of nonlinear physical phenomena. Many methods for seeking solutions of PDEs have been developed, such as the inverse scattering method [1], Hirota's bilinear method [2], symmetry reductions [3], Darboux transformation [4], algebrogeometric method [5], homogeneous balance method [6], and multiple exp-function method [7]. However, except for the soliton-soliton interaction solution, it is very difficult to find interaction solutions among different types of nonlinear excitations with these methods. Recently, a consistent tanh expansion (CTE) method is developed to find interaction solutions between solitons and any other types of solitary waves $[8,9]$. The method has been valid for a lot of integrable systems [10-15]. The method for the nonintegrable nonlinear systems is much less studied. In this paper, we use the CTE method to study two typical nonintegrable systems. The interaction solutions between solitons and any other types of solitary waves for these two equations are obtained via the CTE method. These interaction solutions are completely different from those obtained via other methods [6, 16-18].

The structure of this paper is organized as follows. In Section 2, the CTE approach is developed to the $(3+1)$ dimensional KP equation. The interaction solutions among one soliton and other types of solitary waves such as one resonant soliton solution and cnoidal waves are explicitly given. The interactions between one soliton and cnoidal waves are elastic with phase shifts. According to the above procedure of solving the KP equation, the interaction solutions for the Boussinesq equation are presented in Section 3. The last section is a simple summary and discussion.

\section{CTE Method and Interaction Solutions for (3+1)-Dimensional KP System}

The (3+1)-dimensional Kadomtsev-Petviashvili (KP) equation reads

$$
\left(u_{t}-6 u u_{x}+u_{x x x}\right)_{x}+3 u_{y y}+3 u_{z z}=0,
$$

which has been directly applied in plasma physics. It has also been studied to figure problems of three-dimensional wave structures per se, the wave of collapse of sonic waves, and the self-focusing of the beams of the fast magnetosonic waves propagating in magnetized plasma [19-22].

According to the CTE method, we take the tanh function expansion as the following form by using the leading order analysis [8]:

$$
u=u_{0}+u_{1} \tanh (f)+u_{2} \tanh ^{2}(f),
$$


where $u_{0}, u_{1}, u_{2}$, and $f$ are arbitrary functions of $(t, x, y)$. Vanishing the coefficients of powers of $\tanh ^{6}(f), \tanh ^{5}(f)$, and $\tanh ^{4}(f)$ after substituting (2) into the (3+1)-dimensional KP system (1), we get

$$
\begin{aligned}
& u_{2}=2 f_{x}^{2}, \\
& u_{1}=-2 f_{x x}, \\
& u_{0}=-\frac{4}{3} f_{x}^{2}+\frac{f_{t}+4 f_{x x x}}{6 f_{x}}+\frac{f_{y}^{2}+f_{z}^{2}-f_{x x}^{2}}{2 f_{x}^{2}} .
\end{aligned}
$$

Collecting the coefficients of $\tanh ^{3}(f), \tanh ^{2}(f), \tanh ^{1}(f)$, and $\tanh ^{0}(f)$, we get four overdetermined systems for the field $f$. We omit the expression of these four overdetermined systems since they are very lengthy. According to the CTE approach, we obtain the nonauto-Bäcklund transformation (BT) theorem of (3+1)-dimensional KP equation (1).

Nonauto-BT Theorem 1 . If one finds the solution $f$ to satisfy these four overdetermined systems consistently, then $u$, with

$$
\begin{aligned}
u= & 2 f_{x}^{2} \tanh ^{2}(f)-2 f_{x x} \tanh (f)-\frac{4}{3} f_{x}^{2} \\
& +\frac{f_{t}+4 f_{x x x}}{6 f_{x}}+\frac{f_{y}^{2}+f_{z}^{2}-f_{x x}^{2}}{2 f_{x}^{2}},
\end{aligned}
$$

will be a solution of (3+1)-dimensional KP system (1).

By means of the nonauto-BT Theorem 1, some special interaction solutions among solitons and other kinds of complicated waves can be obtained. We will give some concrete interesting examples in the following.

A quite trivial straight line solution for the overdetermined systems has the form

$$
f=k x+l y+b z+\omega t,
$$

where $k, l, b$, and $\omega$ are the free constants. Substituting the trivial solution (5) into (4), one soliton solution of the $(3+1)$ dimensional KP system yields

$$
\begin{aligned}
u= & 2 k^{2} \tanh ^{2}(k x+l y+b z+\omega t)-\frac{4}{3} k^{2} \\
& +\frac{3 l^{2}+3 b^{2}+\omega k}{6 k^{2}} .
\end{aligned}
$$

The nontrivial solution of the (3+1)-dimensional KP equation is found from the quite trivial solution of (5).

To find the interaction solutions between one soliton and other nonlinear excitations, we can use the solutions with one straight line solution (5) plus undetermined waves for the field $f$. For the interaction solution between one soliton and one resonant soliton solution of the $(3+1)$-dimensional $\mathrm{KP}$ equation, we assume

$$
\begin{aligned}
f= & k x+l y+b z+\omega t \\
& +\frac{1}{2} \ln \left(1+\exp \left(k_{0} x+l_{0} y+b_{0} z+\omega_{0} t\right)\right),
\end{aligned}
$$

where $k_{0}, l_{0}, b_{0}$, and $\omega_{0}$ are arbitrary constants. Substituting expression (7) into overdetermined systems, (7) should be the solution of overdetermined systems with the following relations:

$$
\begin{aligned}
l_{0}= & \frac{k_{0}}{k} l+\frac{\sqrt{4 k^{4} k_{0}^{2}+4 k^{3} k_{0}^{3}+k^{2} k_{0}^{4}-\left(b k_{0}+b_{0} k\right)^{2}}}{k}, \\
\omega_{0}= & \frac{6 b^{2} k_{0}}{k^{2}}+\frac{k_{0} \omega-6 b b_{0}}{k}-4 k_{0}\left(k_{0}^{2}-2 k^{2}-3 k_{0} k\right) \\
& +\frac{6 l \sqrt{4 k^{4} k_{0}^{2}+4 k^{3} k_{0}^{3}+k^{2} k_{0}^{4}-\left(b k_{0}+b_{0} k\right)^{2}}}{k^{2}} .
\end{aligned}
$$

Figure 1 displays the interaction behavior between one soliton and one resonant soliton solution with the parameters selected as $k=1 / 2, b=1, k_{0}=1 / 4, l_{0}=1 / 4, b_{0}=1 / 2$, and $\omega_{0}=1 / 2$. This phenomenon can be observed on the sea surface. Solution (7) is useful for applying in maritime security and coastal engineering.

To find the interaction solutions between one soliton and cnoidal periodic waves, we assume the interaction solution form as

$$
\begin{aligned}
& f=k x+l y+b z+\omega t+F(X), \\
& X=k_{0} x+l_{0} y+b_{0} z+\omega_{0} t .
\end{aligned}
$$

Substituting expression (9) into four overdetermined systems, these four equations will be consistent and the consistent condition is

$$
F_{1, X}^{2}-4 F_{1}^{4}+a_{1} F_{1}^{3}+a_{2} F_{1}^{2}+a_{3} F_{1}+a_{4}=0, \quad F_{X}=F_{1},
$$

where $F_{X}$ and $F_{1, X}$ denote the derivative of $F$ and $F_{1}$ with respect to $X$, respectively, and

$$
\begin{aligned}
a_{1}= & 2 C_{2} k_{0}^{2}-\frac{8 k}{k_{0}}, \\
a_{4}= & \frac{2 k^{2}\left(C_{2} k-C_{1}\right)}{k_{0}}-\frac{k \omega+b^{2}+l^{2}}{k_{0}^{4}} \\
& -\frac{k\left(4 l l_{0}+4 b b_{0}-k \omega_{0}\right)}{k_{0}^{5}}+\frac{5 k^{2}\left(b_{0}^{2}+l_{0}^{2}\right)}{k_{0}^{6}}, \\
a_{2}= & 6 C_{2} k k_{0}-2 C_{1} k_{0}-\frac{4 k^{2}}{k_{0}^{2}}, \\
a_{3}= & 6 C_{2} k^{2}-4 C_{1} k-\frac{\omega}{k_{0}^{3}}+\frac{k \omega_{0}-6 l l_{0}-6 b b_{0}}{k_{0}^{4}} \\
& +\frac{6 k l_{0}^{2}+6 k b_{0}^{2}}{k_{0}^{5}},
\end{aligned}
$$

where $C_{1}$ and $C_{2}$ are arbitrary constants. The general solution of (10) can be written out in terms of Jacobi elliptic functions $[23,24]$. Hence, the solution expressed by (4) is just the explicit interaction solutions between one soliton and cnoidal periodic waves. In the following, we give one special case for this kind of interaction solution. 


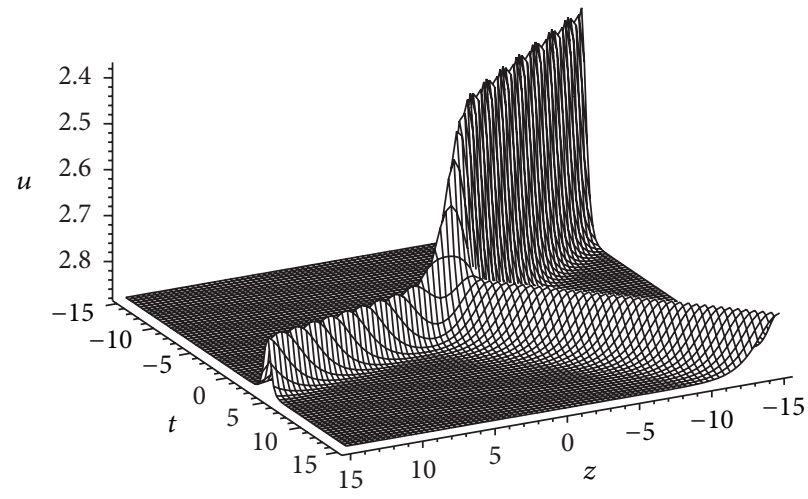

(a)

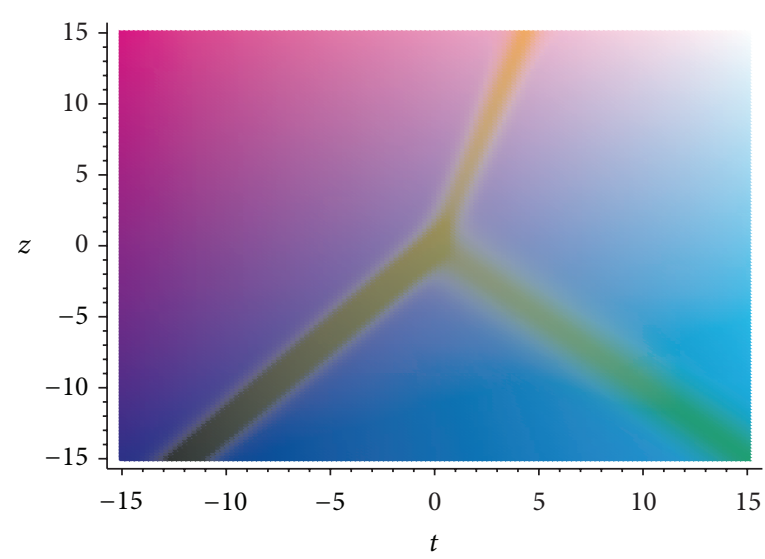

(b)

FIGURE 1: Plot of the interaction solution between one soliton and one resonant soliton solution for field $u$ with the parameters $k=1 / 2, b=1$, $k_{0}=1 / 4, l_{0}=1 / 4, b_{0}=1 / 2$, and $\omega_{0}=1 / 2$. (a) Three-dimensional image at $x=y=0$. (b) The density plot of the corresponding solution.

We take the solution of $(10)$ as

$$
F_{1}=a_{0}+a_{1} \operatorname{sn}\left(a_{2} X, m\right)
$$

Substituting (12) into (10) and vanishing all the coefficients of different powers of the Jacobi elliptic function sn, the interaction solution between one soliton and the cnoidal wave solution of (3+1)-dimensional KP system (1) gives

$$
\begin{aligned}
& u=2\left(k+a_{0} k_{0}+a_{1} k_{0} S\right)^{2} \tanh ^{2}(f)-2 a_{1} a_{2} k_{0}^{2} C D \\
& \cdot \tanh (f)+\left(\frac{1}{6} a_{1}^{2} k_{0}^{4}\left(5 a_{2}^{2} m^{2}-8 a_{1}^{2}\right) S^{4}\right. \\
& +a_{1}^{2}\left(8 a_{0} k_{0}^{3}\left(a_{0} k_{0}+2 k\right)+8 k_{0}^{2} k^{2}\right. \\
& \left.+\frac{1}{6} a_{2}^{2} k_{0}^{4}\left(m^{2}+1\right)-\frac{k_{0} \omega_{0}}{6}-\frac{b_{0}^{2}+l_{0}^{2}}{2}\right) S^{2} \\
& -a_{1}\left(\frac{2}{3} k_{0}^{3}\left(a_{0} k_{0}+k\right)\left(a_{2}^{2} m^{2}+8 a_{0}^{2}+a_{2}^{2}\right)\right. \\
& +k_{0}\left(\frac{16 k^{3}}{3}-\frac{a_{0} \omega_{0}}{3}\right)+16 a_{0} k^{2} k_{0}^{2}-a_{0}\left(l_{0}^{2}+b_{0}^{2}\right) \\
& \left.-l_{0} l-b_{0} b-\frac{\omega k_{0}+\omega_{0} k}{6}\right) S-\frac{4}{3} a_{0}^{4} k_{0}^{4}-\frac{4}{3} k^{4} \\
& +a_{0}^{2}\left(\frac{k_{0} \omega_{0}}{6}+\frac{b_{0}^{2}+l_{0}^{2}}{2}-8 k^{2} k_{0}^{2}\right)+a_{0}\left(\frac{\omega k_{0}+\omega_{0} k}{6}\right. \\
& \left.+b b_{0}+l l_{0}\right)-\frac{16}{3} a_{0} k_{0} k\left(a_{0}^{2} k_{0}^{2}+k^{2}\right)+\frac{b^{2}+l^{2}}{2} \\
& \left.+\frac{k \omega}{6}\right) \cdot\left(k+a_{0} k_{0}+a_{1} k_{0} S\right)^{-2}
\end{aligned}
$$

where $S=\operatorname{sn}\left(a_{2} X, m\right), C=\operatorname{cn}\left(a_{2} X, m\right)$, and $D=\operatorname{dn}\left(a_{2} X, m\right)$ are the usual Jacobian elliptic functions with modulus $m$ and

$$
\begin{aligned}
& a_{1}=-\frac{m a_{2}}{2}, \\
& a_{0}=\frac{C_{2}}{2} k_{0}^{2}+\frac{\Delta}{8} \text {, } \\
& k=-\frac{k_{0}\left(3 C_{2} k_{0}^{2}+\Delta\right)}{4} \text {, } \\
& \Delta=\sqrt{6 C_{2}^{2} k_{0}^{4}+16 C_{1} k_{0}+8 a_{2}^{2} m^{2}+8 a_{2}^{2}}, \\
& l=-\frac{3 C_{2} l_{0} k_{0}^{2}+l_{0} \Delta}{4}+\frac{1}{16}\left(64 m^{2} a_{2}^{4} k_{0}^{4}\right. \\
& -4 a_{2}^{2} k_{0}^{4}\left(m^{2}+1\right)\left(2 C_{2} k_{0}^{2}+\Delta\right)^{2} \\
& +\left(2 C_{2}^{2} k_{0}^{6}+2 C_{2} \Delta k_{0}^{4}+\frac{1}{2} \Delta^{2} k_{0}^{2}\right)^{2} \\
& \left.-\left(12 C_{2} b_{0} k_{0}^{2}+4 b_{0} \Delta+16 b\right)^{2}\right)^{1 / 2} \text {, } \\
& \omega=\left(\frac{C_{1} k_{0}^{4}}{2}+\frac{9 C_{2}^{2} k_{0}^{7}}{16}-\frac{k_{0} \omega_{0}+6 l_{0}^{2}+6 b_{0}^{2}}{4 k_{0}}\right) \Delta \\
& +\frac{11 C_{2}^{3} k_{0}^{9}}{8}+3 C_{1} C_{2} k_{0}^{6} \\
& +\frac{C_{2} k_{0}\left(4 a_{2}^{2} k_{0}^{4}\left(1+m^{2}\right)-18 b_{0}^{2}-3 k_{0} \omega_{0}-18 l_{0}^{2}\right)}{4} \\
& -\frac{6\left(b b_{0}+l l_{0}\right)}{k_{0}}
\end{aligned}
$$

It is remarkable that solution (13) describes interaction between one soliton and the cnoidal wave. Figure 2 plots a 
dark soliton coupled to a cnoidal wave background with the parameters $k_{0}=1, l_{0}=1, b_{0}=1 / 4, \omega_{0}=1, b=1 / 4$, $a_{2}=1, m=0.3, C_{1}=1$, and $C_{2}=0.75$. Figures $2(\mathrm{a})$ and 2(b) exhibit the wave interaction structure for $(3+1)$ dimensional KP equation solution (13) at $x=y=z=0$ and $x=y=t=0$, respectively. Figure $2(\mathrm{c})$ is threedimensional view of corresponding solution (13). Figure 2(d) demonstrates the interaction behavior between a soliton and every peak of the periodic wave. It is easy to observe that the interaction between a soliton and every peak of the cnoidal periodic wave is elastic except phase shifts. In the ocean, there are some typical nonlinear waves such as interaction solutions between solitons and cnoidal periodic waves [23]. We introduce the interaction solutions which may be useful for studying the ocean waves.

\section{CTE Method and Interaction Solutions for $(2+1)$-Dimensional Boussinesq System}

The (2+1)-dimensional Boussinesq equation reads

$$
u_{t t}+6 u_{x}^{2}+6 u u_{x x}-u_{x x}-u_{x x x x}-u_{y y}=0 .
$$

It was derived by combining the classical Boussinesq equation with the weak dependence on the second spatial dimension [25]. It can be used to describe the propagation of gravity waves on water surface, in particular the head-on collision of oblique waves $[25,26]$.

According to the same as the above-mentioned steps, the tanh function expansion has the form [8]

$$
u=u_{0}+u_{1} \tanh (f)+u_{2} \tanh ^{2}(f)
$$

where $u_{0}, u_{1}, u_{2}$, and $f$ are arbitrary functions of $(t, x, y)$. Vanishing the coefficients of powers of $\tanh ^{6}(f), \tanh ^{5}(f)$, and $\tanh ^{4}(f)$ after substituting (16) into the Boussinesq system (15), we get

$$
\begin{aligned}
& u_{2}=2 f_{x}^{2}, \\
& u_{1}=-2 f_{x x}, \\
& u_{0}=\frac{1}{6}-\frac{4}{3} f_{x}^{2}+\frac{2 f_{x x x}}{3 f_{x}}+\frac{f_{y}^{2}-f_{t}^{2}-3 f_{x x}^{2}}{6 f_{x}^{2}} .
\end{aligned}
$$

Gathering the coefficients of $\tanh ^{3}(f), \tanh ^{2}(f), \tanh ^{1}(f)$, and $\tanh ^{0}(f)$, we get four overdetermined systems for the field $f$. The following nonauto-BT theorem for $(2+1)$ dimensional Boussinesq equation (15) is obtained with above calculation.

Nonauto-BT Theorem. If one finds the solution $f$ to satisfy four overdetermined systems consistently, then $u$, with

$$
\begin{aligned}
u= & 2 f_{x}^{2} \tanh ^{2}(f)-2 f_{x x} \tanh (f)+\frac{1}{6}-\frac{4}{3} f_{x}^{2} \\
& +\frac{2 f_{x x x}}{3 f_{x}}+\frac{f_{y}^{2}-f_{t}^{2}-3 f_{x x}^{2}}{6 f_{x}^{2}}
\end{aligned}
$$

will be a solution of $(2+1)$-dimensional Boussinesq system (15). To find the interaction solutions between one soliton and cnoidal periodic waves, we assume the interaction solution form as

$$
\begin{aligned}
& f=k x+l y+\omega t+F(X), \\
& X=k_{0} x+l_{0} y+\omega_{0} t,
\end{aligned}
$$

where $k_{0}, l_{0}, \omega_{0}, k, l$, and $\omega$ are all the free constants. Substituting (19) into overdetermined systems, we obtain an equation about $F_{1}(X)$ as

$$
F_{1, X}^{2}-4 F_{1}^{4}+a_{1} F_{1}^{3}-a_{2} F_{1}^{2}-a_{3} F_{1}-a_{4}=0, \quad F_{X}=F_{1},
$$

where $F_{X}$ and $F_{1, X}$ indicate the derivative of $F$ and $F_{1}$ with respect to $X$, respectively, and

$$
\begin{aligned}
a_{1}= & 2 C_{2} k_{0}^{2}-\frac{8 k}{k_{0}}, \\
a_{4}= & \frac{2 k^{2}\left(C_{1}-C_{2} k\right)}{k_{0}}+\frac{l^{2}-\omega^{2}}{3 k_{0}^{4}}+\frac{4 k\left(l l_{0}-\omega \omega_{0}\right)}{3 k_{0}^{5}} \\
& -\frac{5 k^{2}\left(l_{0}^{2}-\omega_{0}^{2}\right)}{3 k_{0}^{6}}, \\
a_{2}= & 2 k_{0}\left(C_{1}-3 C_{2} k\right)+\frac{4 k^{2}}{k_{0}^{2}}, \\
a_{3}= & 2 k\left(2 C_{1}-3 C_{2} k\right)+\frac{2\left(l l_{0}-\omega \omega_{0}\right)}{k_{0}^{4}}-\frac{2 k\left(l_{0}^{2}-\omega_{0}^{2}\right)}{k_{0}^{5}},
\end{aligned}
$$

where $C_{1}$ and $C_{2}$ are arbitrary constants. We also give one special case to show interaction solutions between one soliton and cnoidal periodic waves.

We assume the solution of (20) as

$$
F_{1}=a_{0}+a_{1} \operatorname{sn}\left(a_{2} X, m\right) .
$$

Substituting (22) into (20) and vanishing all the coefficients of different powers of the Jacobian elliptic function sn, the interaction solution between one soliton and the cnoidal wave solution of (2+1)-dimensional Boussinesq system (15) gives

$$
\begin{aligned}
u= & 2\left(k+a_{0} k_{0}+a_{1} k_{0} S\right)^{2} \tanh ^{2}(f)-2 k_{0}^{2} a_{1} a_{2} C D \\
& \cdot \tanh (f)+\left(a_{1}^{2} k_{0}^{4}\left(8 a_{1}^{2}-5 a_{2}^{2} m^{2}\right) s^{4}\right. \\
& +a_{1}^{2}\left(48 a_{0} k_{0}^{3}\left(a_{0} k_{0}+2 k\right)+a_{2}^{2} k_{0}^{4}\left(m^{2}+1\right)\right. \\
& \left.+k_{0}^{2}\left(48 k^{2}-1\right)+\omega_{0}^{2}-l_{0}^{2}\right) s^{2} \\
& +2 a_{1}\left(2 a_{2}^{2} k_{0}^{3}\left(m^{2}+1\right)\left(a_{0} k_{0}+k\right)\right. \\
& +16 a_{0}^{2} k_{0}^{3}\left(a_{0} k_{0}+3 k\right)+a_{0} k_{0}^{2}\left(48 k^{2}-1\right) \\
& \left.+k_{0} k\left(16 k^{2}-1\right)+a_{0}\left(\omega^{2}-l^{2}\right)+\omega \omega_{0}-l l_{0}\right) S \\
& +k_{0}^{4}\left(8 a_{0}^{4}+3 a_{1}^{2} a_{2}^{2}\right)+a_{0}^{2} k_{0}^{2}\left(48 k^{2}-1\right)+8 k^{4} \\
& +32 k a_{0}^{3} k_{0}^{3}+2 a_{0} k k_{0}\left(16 k^{2}-1\right)+a_{0}^{2}\left(\omega^{2}-l^{2}\right)
\end{aligned}
$$




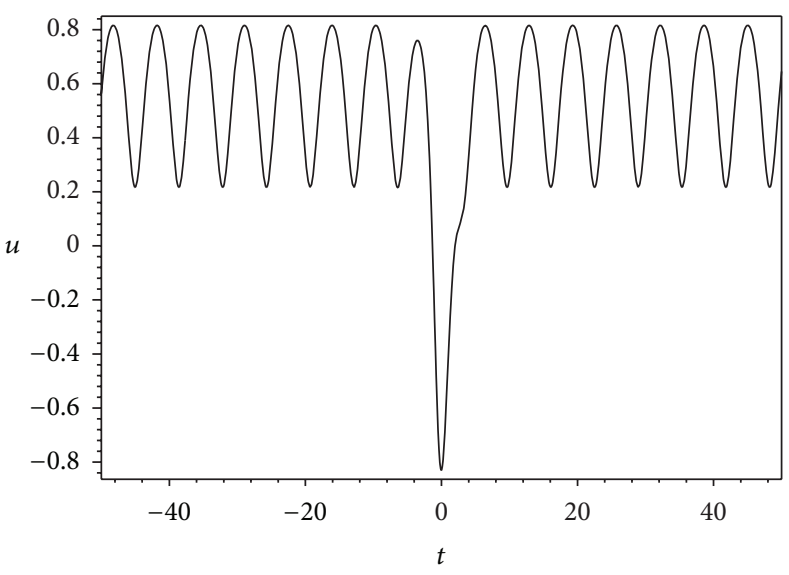

(a)

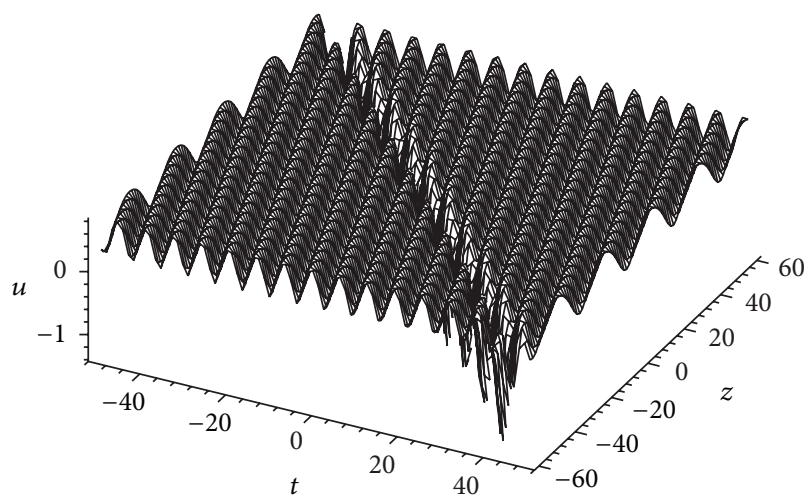

(c)

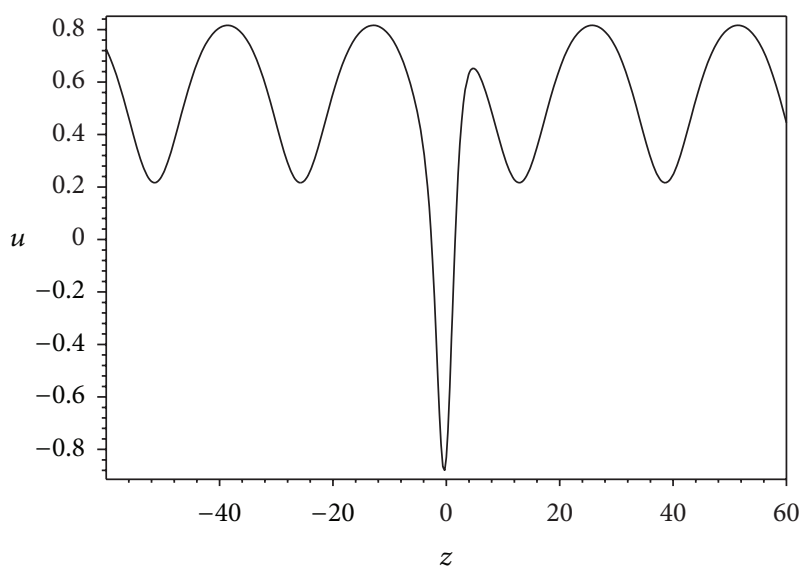

(b)

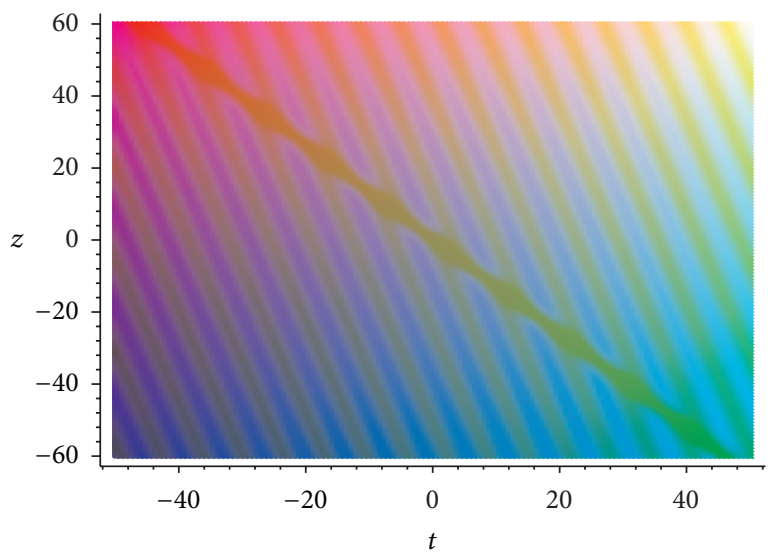

(d)

FIGURE 2: Plot of one soliton on the periodic cnoidal wave background expressed by (13). (a) The wave propagation pattern of the wave along $t$ axis at $x=y=z=0$. (b) The soliton-cnoidal wave structure at $x=y=t=0$. (c) The three-dimensional view at $x=y=0$. (d) The density plot of the corresponding solution.

$$
\begin{aligned}
& \left.+2 a_{0}\left(\omega \omega_{0}-l l_{0}\right)+\omega^{2}-l^{2}-k^{2}\right) \cdot\left(6 \left(k+a_{0} k_{0}\right.\right. \\
& \left.\left.+a_{1} k_{0} S\right)^{2}\right)^{-1}
\end{aligned}
$$

where $S=\operatorname{sn}\left(a_{2} X, m\right), C=\operatorname{cn}\left(a_{2} X, m\right)$, and $D=\operatorname{dn}\left(a_{2} X, m\right)$ are the usual Jacobian elliptic functions with modulus $m$ and

$$
\begin{aligned}
a_{1}= & -\frac{m a_{2}}{2}, \\
a_{0}= & \frac{C_{1}}{2} k_{0}^{2}+\frac{\Delta}{8}, \\
k= & -\frac{k_{0}\left(3 C_{1} k_{0}^{2}+\Delta\right)}{4}, \\
\Delta= & \sqrt{6 C_{1}^{2} k_{0}^{4}+16 C_{2} k_{0}+8 a_{2}^{2} m^{2}+8 a_{2}^{2}}, \\
l= & \frac{l_{0} k_{0}^{4}\left(2 C_{2} k_{0}^{2}+\Delta\right)^{3}}{64\left(l_{0}^{2}-\omega_{0}^{2}\right)}-\frac{l_{0} k_{0}^{4}\left(2 C_{2} k_{0}^{2}+\Delta\right)\left(m^{2}+1\right)}{8\left(l_{0}^{2}-\omega_{0}^{2}\right)} \\
& -\frac{l_{0}}{4} \Delta-\frac{3 C_{2} l_{0} k_{0}^{2}}{4}+\frac{k_{0}^{2}\left|\omega_{0}\right|}{\left(l_{0}^{2}-\omega_{0}^{2}\right)}\left(a_{2}^{4} k_{0}^{4}\left(m^{2}+1\right)^{2}\right.
\end{aligned}
$$




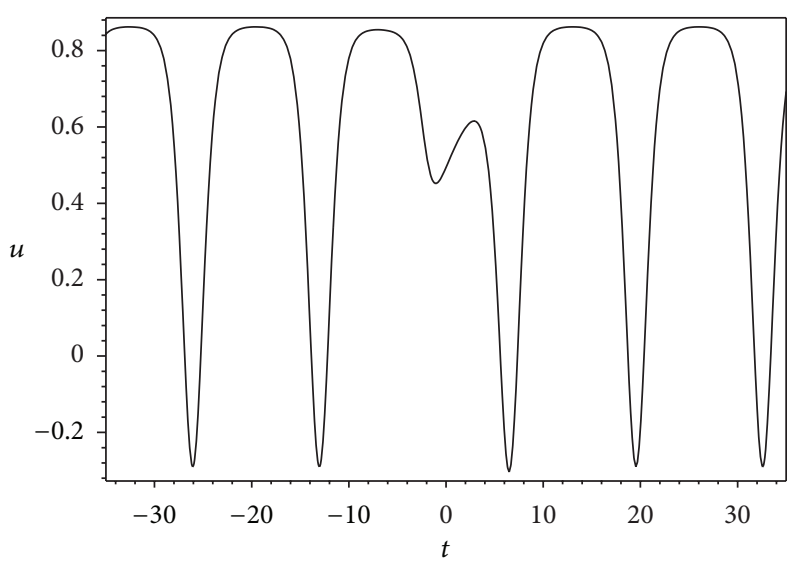

(a)

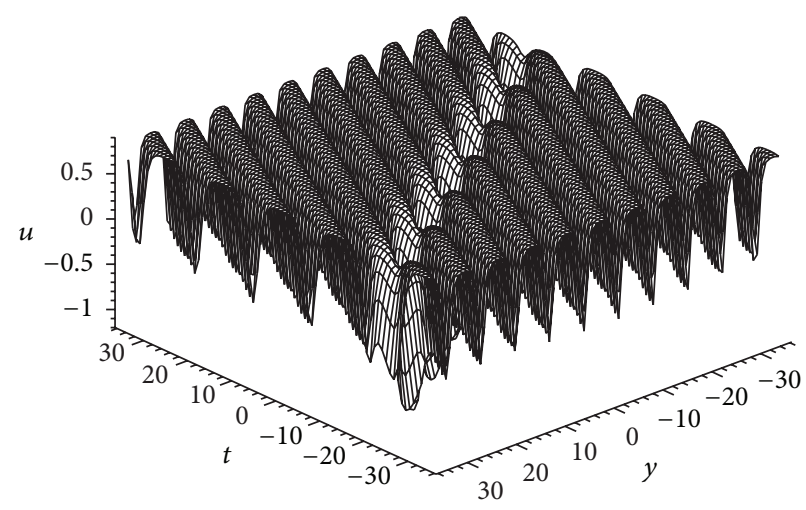

(c)

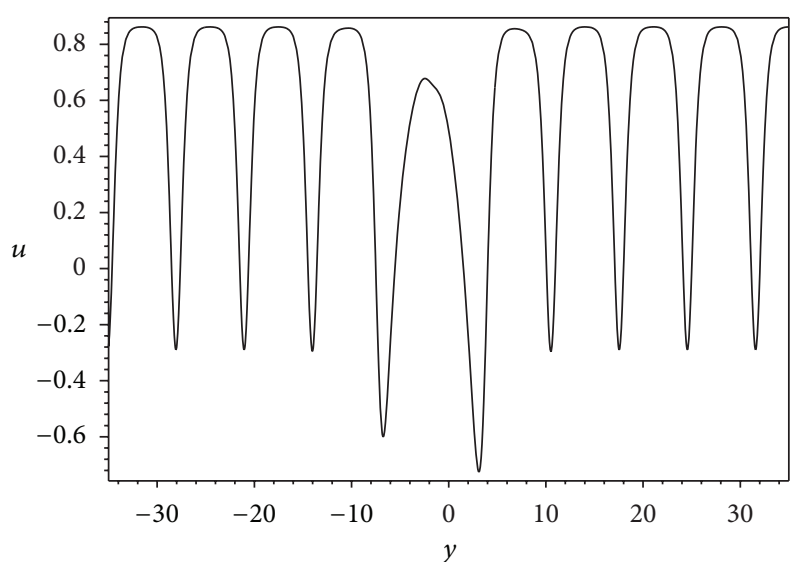

(b)

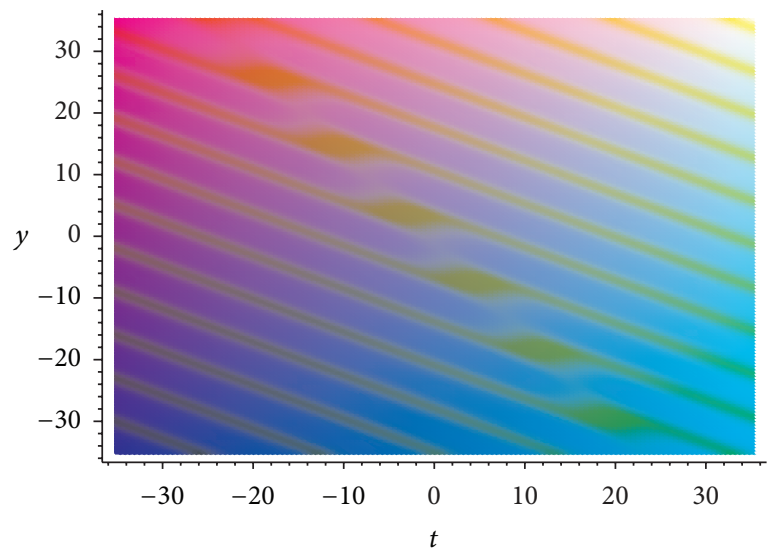

(d)

Figure 3: Plot of one soliton between the periodic cnoidal wave interaction solution expressed by (23) of the Boussinesq equation. (a) The wave propagation pattern of the wave along $t$ axis at $x=y=0$. (b) The soliton-cnoidal wave structure at $x=t=0$. (c) The three-dimensional view at time $x=0$. (d) The density plot of the corresponding solution.

Figure 3 is a special plot of this interaction solution with the parameters $k_{0}=0.8, l_{0}=1.3, \omega_{0}=0.7, a_{2}=1$, $m=0.9, C_{1}=1$, and $C_{2}=0.75$. Figures $3(\mathrm{a})$ and $3(\mathrm{~b})$ exhibit the wave interaction structure for $(2+1)$-dimensional Boussinesq equation solution (23) at $x=y=0$ and $x=$ $t=0$, respectively. Figure $3(\mathrm{c})$ is three-dimensional view of corresponding solution (23). Figure 3(d) shows that the interaction between soliton and the cnoidal wave (every peak of the cnoidal wave) is elastic except for phase shifts.

\section{Conclusions}

In summary, the (3+1)-dimensional $\mathrm{KP}$ and $(2+1)$ dimensional Boussinesq equations are studied by means of the CTE method. A nonauto-BT theorem for these two systems is given with the CTE method. With the help of the nonauto-BT theorem, the interaction solutions between one soliton and cnoidal waves are obtained for these two systems. The interaction between one soliton and every peak of the cnoidal periodic wave is elastic with phase shift. Besides the interaction solution between one soliton and one resonant soliton solution for $(3+1)$-dimensional $\mathrm{KP}$ equation is also derived with the nonauto-BT theorem. The interaction solutions for these two nonintegrable equations were not found before. It may be useful for studying the ocean waves. In the meanwhile, other methods such as the truncated Painleve expansion [8], Darboux transformation $[23,24,27,28]$, and Bäcklund transformation [29] related nonlocal symmetries have been also used to obtain the interaction solutions among solitons and other nonlinear excitations. The details on these interaction solutions for other nonlinear systems are worthy of further study.

\section{Conflict of Interests}

The authors declare that there is no conflict of interests regarding the publication of this paper.

\section{Acknowledgments}

This work was partially supported by the National Natural Science Foundation of China under Grant nos. 11305106, 11275129, and 11405110 and the Natural Science Foundation of Zhejiang Province of China under Grant no. LQ13A050001. 


\section{References}

[1] C. S. Gardner, J. M. Greene, M. D. Kruskal, and R. M. Miura, "Method for solving the Korteweg-deVries equation," Physical Review Letters, vol. 19, no. 19, pp. 1095-1097, 1967.

[2] R. Hirota, The Direct Method in Soliton Theory, Cambridge University Press, Cambridge, UK, 2004.

[3] J. Olver P, Application of Lie Groups to Differential Equation, Graduate Texts in Mathematics, Springer, New York, NY, USA, 2nd edition, 1993.

[4] V. B. Matveev and M. A. Salle, Darboux Transformation and Solitons, Springer, Berlin, Germany, 1991.

[5] E. Belokolos, A. Bobenko, V. Enol'skij, A. Its, and V. Matveev, Algebro-Geometrical Approach to Nonlinear Integrable Equations, Springer, Berlin, Germany, 1994.

[6] Y. Chen, Z. Yan, and H. Zhang, "New explicit solitary wave solutions for $(2+1)$-dimensional Boussinesq equation and $(3+1)$-dimensional KP equation," Physics Letters, Section A: General, Atomic and Solid State Physics, vol. 307, no. 2-3, pp. 107113, 2003.

[7] W.-X. Ma and Z. Zhu, "Solving the $(3+1)$-dimensional generalized KP and BKP equations by the multiple exp-function algorithm," Applied Mathematics and Computation, vol. 218, no. 24, pp. 11871-11879, 2012.

[8] X. N. Gao, S. Y. Lou, and X. Y. Tang, "Bosonization, singularity analysis, nonlocal symmetry reductions and exact solutions of supersymmetric KdV equation," Journal of High Energy Physics, vol. 2013, no. 5, article 029, 2013.

[9] S. Y. Lou, "Consistent Riccati expansion and solvability," Studies in Applied Mathematics, vol. 134, no. 3, pp. 372-402, 2015.

[10] S. Y. Lou, X. P. Cheng, and X. Y. Tang, "Dressed dark solitons of the defocusing nonlinear Schrödinger equation," Chinese Physics Letters, vol. 31, no. 7, Article ID 070201, 2014.

[11] C.-L. Chen and S.-Y. Lou, "CTE solvability and exact solution to the Broer-Kaup system," Chinese Physics Letters, vol. 30, no. 11, Article ID 110202, 4 pages, 2013.

[12] B. Ren, X.-Z. Liu, and P. Liu, "Nonlocal symmetry reductions, CTE method and exact solutions for higher-order KdV equation," Communications in Theoretical Physics, vol. 63, no. 2, pp. 125-128, 2015.

[13] Y.-H. Wang and H. Wang, "Symmetry analysis and CTE solvability for the (2+1)-dimensional Boiti-Leon-Pempinelli equation," Physica Scripta, vol. 89, no. 12, Article ID 125203, 2014.

[14] D. Yang, S.-Y. Lou, and W.-F. Yu, "Interactions between solitons and cnoidal periodic waves of the Boussinesq equation," Communications in Theoretical Physics, vol. 60, no. 4, pp. 387-390, 2013.

[15] B. Ren, "Interaction solutions for mKP equation with nonlocal symmetry reductions and CTE method," Physica Scripta, vol. 90, no. 6, Article ID 065206, 2015.

[16] M. Senthilvelan, "On the extended applications of homogenous balance method," Applied Mathematics and Computation, vol. 123, no. 3, pp. 381-388, 2001.

[17] H. Zhang, B. Tian, H.-Q. Zhang et al., "Periodic wave solutions for $(2+1)$-dimensional Boussinesq equation and $(3+1)$ dimensional Kadomtsev-Petviashvili equation," Communications in Theoretical Physics, vol. 50, no. 5, pp. 1169-1176, 2008.

[18] Q. Zhao, S.-K. Liu, and Z.-T. Fu, "Exact periodic-wave solutions for $(2+1)$-dimensional Boussinesq equation and $(3+$ 1)-dimensional KP equation," Communications in Theoretical Physics, vol. 42, no. 2, pp. 239-241, 2004.
[19] E. A. Kuznetsov and S. L. Musher, "Effect of collapse of sound waves on the structure of collisionless shock waves in a magnetized plasma," Zhurnal Eksperimental'noi i Teoreticheskoi Fiziki, vol. 91, no. 5, pp. 1605-1619, 1986.

[20] T. Alagesan, A. Uthayakumar, and K. Porsezian, "Painlevé analysis and Bäcklund transformation for a three-dimensional Kadomtsev-Petviashvili equation," Chaos, Solitons \& Fractals, vol. 8, no. 6, pp. 893-895, 1997.

[21] V. I. Karpman and V. Y. Belashov, "Evolution of threedimensional nonlinear pulses in weakly dispersive media," Physics Letters A, vol. 154, no. 3-4, pp. 140-144, 1991.

[22] Z. Yan, "Multiple solution profiles to the higher-dimensional Kadomtsev-Petviashvilli equations via Wronskian determinant," Chaos, Solitons and Fractals, vol. 33, no. 3, pp. 951-957, 2007.

[23] X.-R. Hu, S.-Y. Lou, and Y. Chen, "Explicit solutions from eigenfunction symmetry of the Korteweg-de Vries equation," Physical Review E, vol. 85, no. 5, Article ID 056607, 8 pages, 2012.

[24] X.-P. Cheng, S. Y. Lou, C.-L. Chen, and X.-Y. Tang, "Interactions between solitons and other nonlinear Schrödinger waves," Physical Review E-Statistical, Nonlinear, and Soft Matter Physics, vol. 89, no. 4, Article ID 043202, 2014.

[25] R. S. Johnson, "A two-dimensional Boussinesq equation for water waves and some of its solutions," Journal of Fluid Mechanics, vol. 323, no. 9, pp. 65-78, 1996.

[26] H.-Q. Zhang, X.-H. Meng, J. Li, and B. Tian, "Soliton resonance of the (2+1)-dimensional Boussinesq equation for gravity water waves," Nonlinear Analysis: Real World Applications, vol. 9, no. 3, pp. 920-926, 2008.

[27] J. Chen, X. Xin, and Y. Chena, "Nonlocal symmetries of the hirota-satsuma coupled korteweg-de vries system and their applications: exact interaction solutions and integrable hierarchy," Journal of Mathematical Physics, vol. 55, no. 5, Article ID 053508, 2014.

[28] X.-P. Cheng, C.-L. Chen, and S. Y. Lou, "Interactions among different types of nonlinear waves described by the KadomtsevPetviashvili equation," Wave Motion, vol. 51, no. 8, pp. 12981308, 2014.

[29] S. Y. Lou, X. Hu, and Y. Chen, "Nonlocal symmetries related to Bäcklund transformation and their applications," Journal of Physics A: Mathematical and Theoretical, vol. 45, no. 15, Article ID 155209, 2012. 


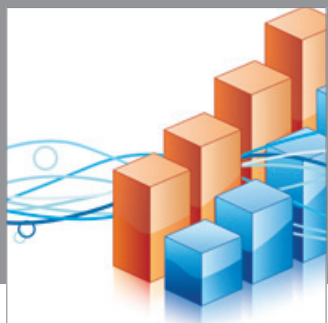

Advances in

Operations Research

mansans

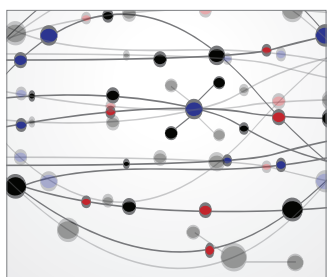

The Scientific World Journal
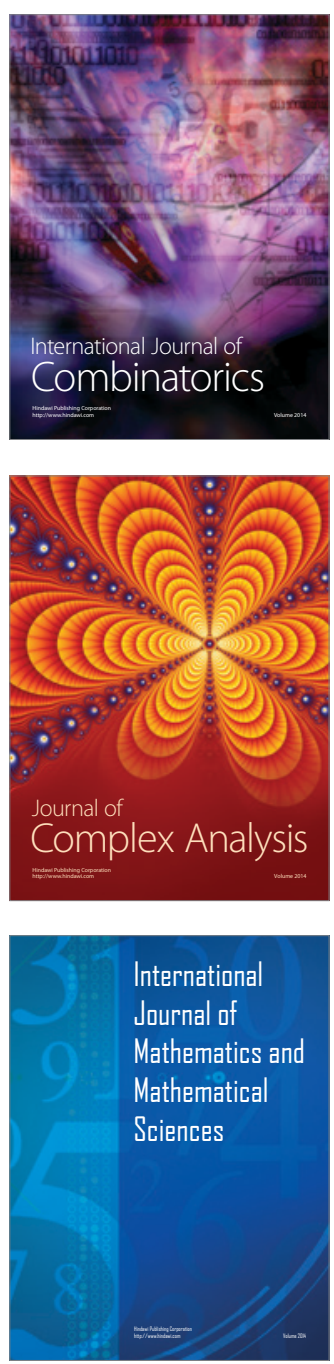
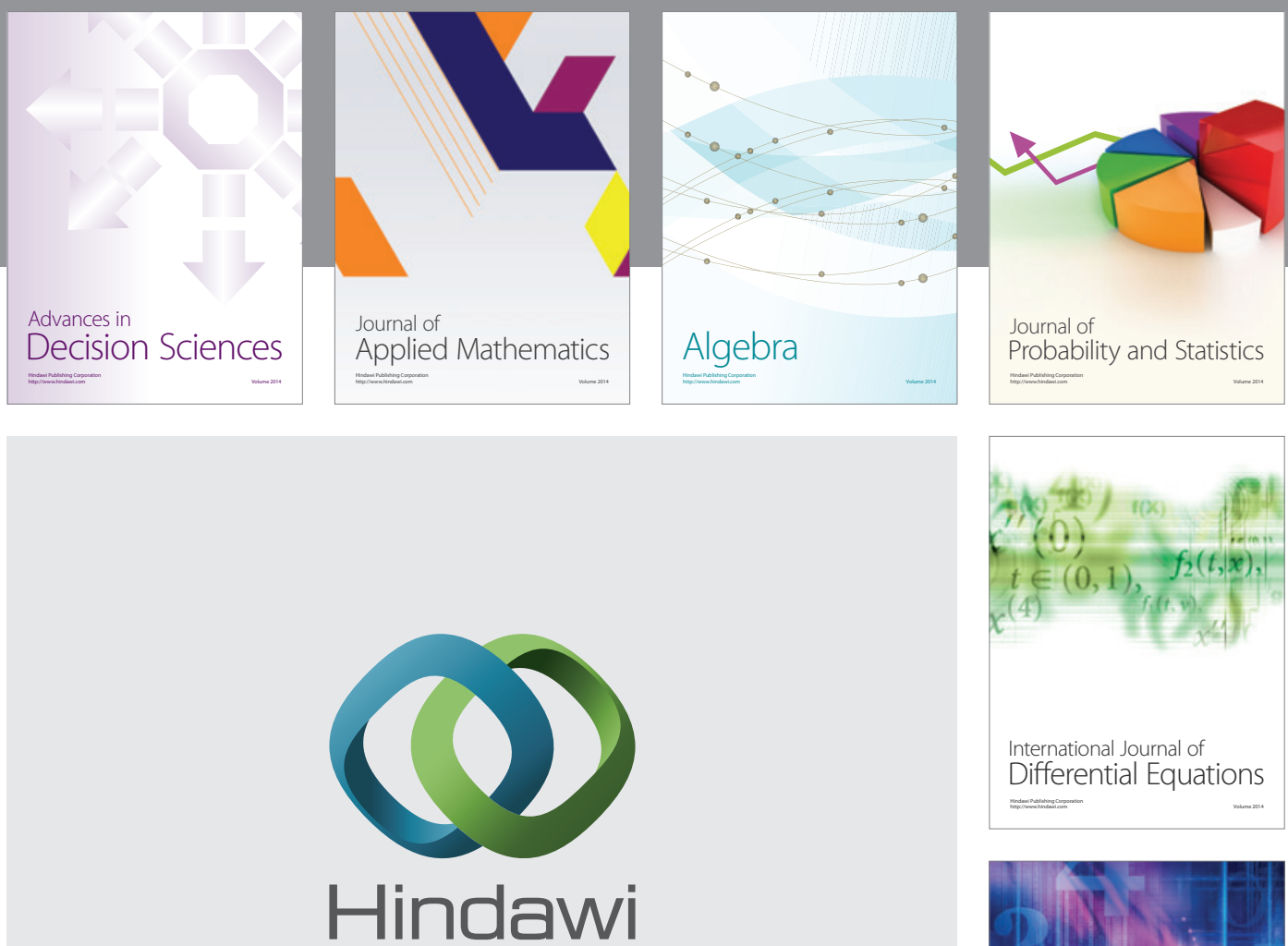

Submit your manuscripts at http://www.hindawi.com
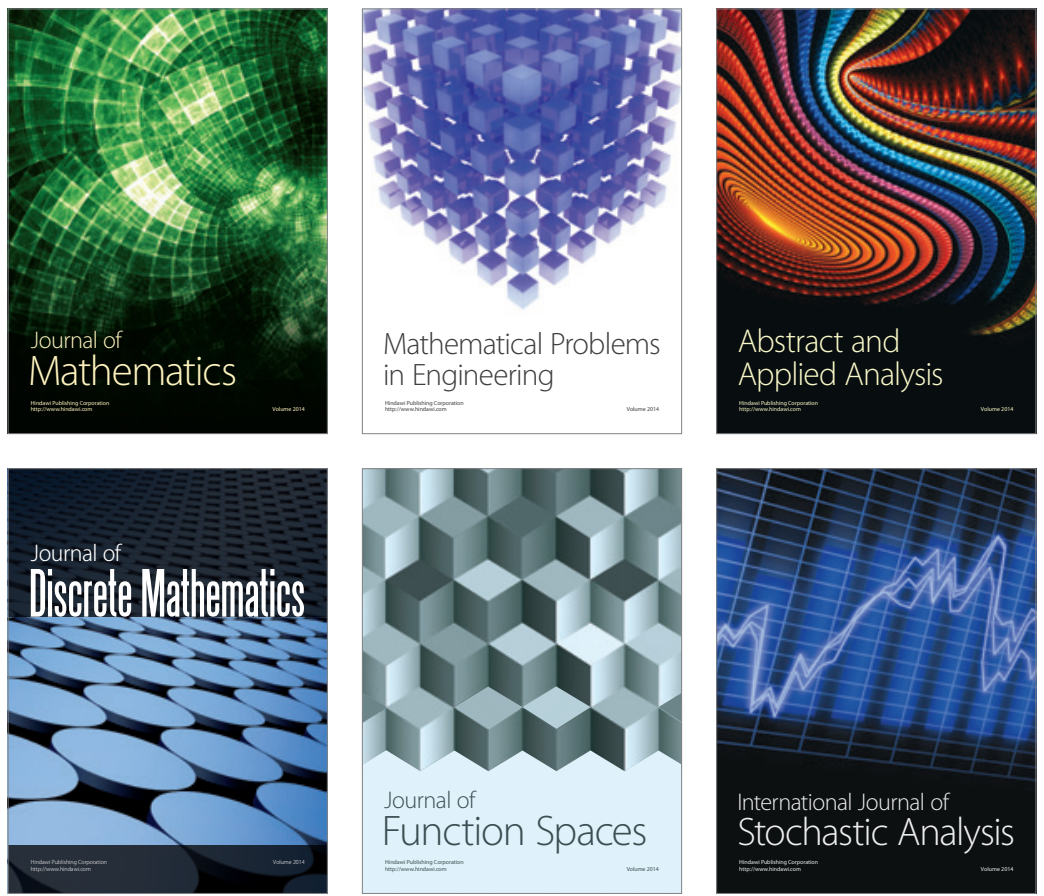

Journal of

Function Spaces

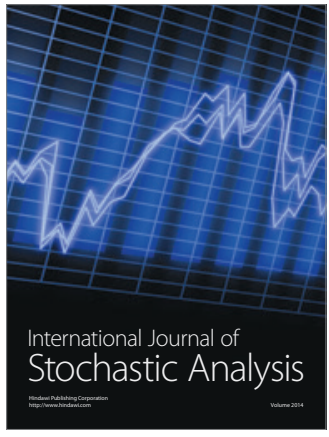

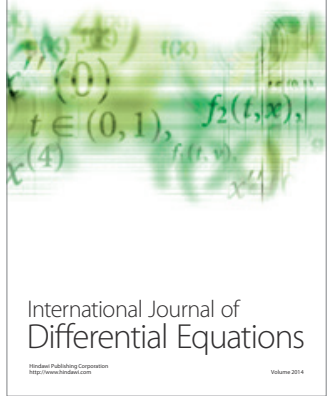
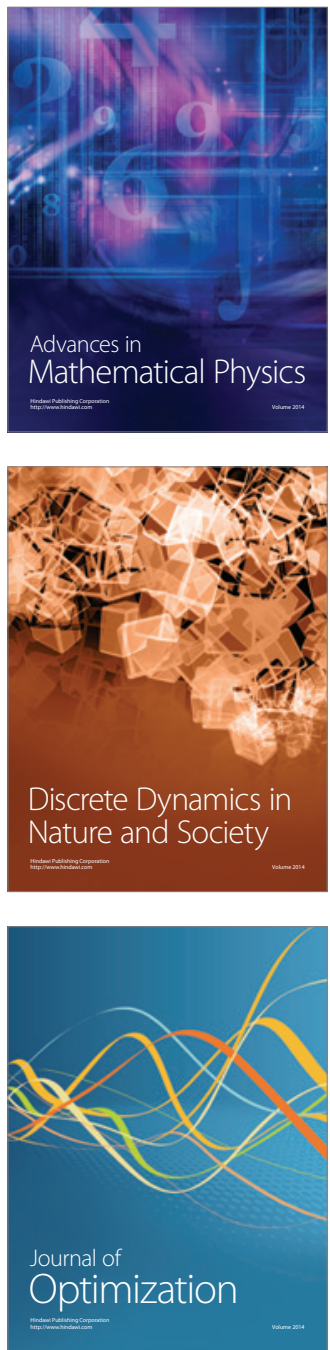